\title{
Lycorine inhibits cell proliferation and migration by inhibiting ROCK1/cofilin-induced actin dynamics in HepG2 hepatoblastoma cells
}

\author{
WUYI LIU*, QIAN ZHANG*, QIN TANG, CHANGPENG HU, JINGBIN HUANG, \\ YALI LIU, YANYILU, QING WANG, GUOBING LI and RONG ZHANG \\ Department of Pharmacy, Xinqiao Hospital, Army Medical University, Chongqing 400037, P.R. China
}

Received January 23, 2018; Accepted July 24, 2018

DOI: $10.3892 / o r .2018 .6609$

\begin{abstract}
Lycorine, a natural alkaloid extracted from the Amaryllidaceae plant family, has been reported to exhibit anti-cancer effects in various types of cancer cells. However, the molecular mechanisms through which lycorine exhibits anti-hepatoblastoma activity are unclear. In the present study, the inhibitory effects of lycorine on the proliferation and migration of HepG2 hepatoblastoma cells were investigated. Lycorine inhibited the proliferation of HepG2 cells in a dose-dependent manner by inducing cell cycle arrest at the G2/M phase, via downregulation of cyclin A, cyclin B1 and cyclin dependent kinase 1 . Additionally, wound healing and Transwell assays revealed that treatment with lycorine resulted in a decrease in the migratory ability of HepG2 cells. Also, treatment with lycorine decreased the expression levels of matrix metalloproteinase (MMP)-9 and MMP-2. Furthermore, lycorine induced the cleavage/activation of Rho associated coiled-coil containing protein kinase 1 (ROCK1) and the downregulation of cofilin, accompanied by an increase in polymerized filamentous actin and a loss of depolymerized globular actin. Furthermore, pre-incubation of cells with Y-27632, a specific ROCK1 inhibitor, markedly attenuated lycorine-induced anti-proliferative and anti-migration effects. Taken together, the results demonstrated that lycorine inhibited the proliferation and migration of HepG2 cells by suppressing ROCK1/cofilin-induced actin dynamics, which suggests that lycorine has the potential to be developed into a novel drug for hepatoblastoma treatment.
\end{abstract}

Correspondence to: Professor Rong Zhang or Dr Guobing Li, Department of Pharmacy,Xinqiao Hospital, Army Medical University, 83 Xinqiao Road, Chongqing 400037, P.R. China

E-mail: xqpharmacylab@126.com

E-mail:rgwlsb@126.com

${ }^{*}$ Contributed equally

Key words: lycorine, actin dynamics, Rho associated coiled-coil containing protein kinase 1 , cofilin, hepatoblastoma

\section{Introduction}

Hepatoblastoma, the most commonly diagnosed malignant pediatric liver tumor, is frequently diagnosed in the first 3 years of life. In recent years, the combination of surgery and chemotherapy has improved the prognosis of patients with hepatoblastoma (1). Furthermore, chemotherapeutic agents, including cisplatin, have been applied in therapeutic strategies for hepatoblastoma $(2,3)$. However, conventional chemotherapy agents frequently have limited clinical applications due to the adverse side effects and drug resistance acquired following long-term use. Consequently, it is vital to develop safe and affordable alternative therapeutic agents for the treatment of hepatoblastoma.

Recently, naturally occurring compounds have been valued as potential anticancer therapies due to their safety and efficacy. Additionally, the majority of clinical chemotherapeutic drugs have an alkaloid structure, suggesting that alkaloids are important antitumor agent candidates. Lycorine, a crude alkaloid extracted from Amaryllidaceae genera, is reported to have antimalarial, antiviral and anti-inflammatory properties (4-7). Notably, lycorine is at least 15 -fold more effective against cancer cells compared with normal cells, suggesting that lycorine is a selective anti-tumor compound (8). Multiple molecular mechanisms have been reported to be involved in the anticancer effects of lycorine. Inducing apoptosis of cancer cells has a pivotal role among these mechanisms. For example, lycorine induces apoptosis of A549 cells via the adenosine monophosphate-activated protein kinase/serine/threonine-protein kinase mTOR/S6 kinase signaling pathway (9), and lycorine induces apoptosis of bladder cancer T24 cells by inhibiting protein kinase $\mathrm{B}$ phosphorylation and activating the intrinsic apoptotic cascade (10). However, increasing evidence has emphasized that lycorine also inhibits cancer cell proliferation and migration (11). Thus, it is important to examine the molecular mechanisms underlying the anti-proliferative and anti-migration effects.

In the present study, the HepG2 hepatoblastoma cell line was used to investigate the inhibitory effects of lycorine on cell proliferation and migration. Lycorine inhibited the proliferation of HepG2 cells and induced cell cycle arrest at the G2/M phase. Additionally, lycorine inhibited the migration 
of HepG2 cells. Furthermore, mechanistic analyses revealed that lycorine inhibited HepG2 cell proliferation and migration through suppression of Rho associated coiled-coil containing protein kinase 1 (ROCK1)/cofilin-induced actin dynamics. Furthermore, pre-incubation of cells with Y-27632, a specific ROCK1 inhibitor, significantly attenuated the anti-proliferative and anti-migration effects of lycorine. Additionally, Y-27632 attenuated lycorine-induced cofilin downregulation and $\mathrm{G} 2 / \mathrm{M}$ phase cell cycle arrest. These results suggested that lycorine may be useful as a promising agent for treating hepatoblastoma.

\section{Materials and methods}

Cells and antibodies. Lycorine (cat. no. A0415) was purchased from Chengdu Must Biotechnology Co., Ltd. (Chengdu, China) and dissolved in PBS as a stock solution. Y-27632 (cat. no. S1049) was obtained from Selleck Chemicals (Houston, TX, USA). Antibodies against cyclin A (cat. no. sc-751; 1:500), cyclin B1 (cat. no. sc-752; 1:500), cyclin dependent kinase 1 (cdc2; cat. no. sc-8395; 1:1,000) and GAPDH (cat. no. sc-51905; 1:10,000) were purchased from Santa Cruz Biotechnology, Inc. (Dallas, TX, USA); antibodies against cofilin (cat. no. ab42824; 1:2,000) and ROCK1 (cat. no. ab45171; 1:1,000) were from Abcam (Cambridge, MA, USA); and antibodies against matrix metalloproteinase (MMP)-9 (cat. no. 13667; 1:1,000) and MMP-2 (cat. no. 40994; 1:1,000) were from Cell Signaling Technology, Inc. (Danvers, MA, USA).

Cell culture. The human HepG2 hepatoblastoma cell line was obtained from the American Type Culture Collection (Manassas, VA, USA). Cells were cultured in Dulbecco's modified Eagle's medium (DMEM; cat. no. PM150212; Procell Life Science \& Technology Co., Ltd., Wuhan, China) supplemented with $10 \%$ fetal bovine serum (FBS; Gibco; Thermo Fisher Scientific, Inc., Waltham, MA, USA) and cultured in a $37^{\circ} \mathrm{C}$ incubator with a humidified atmosphere of $5 \% \mathrm{CO}_{2}$. Once cells were adhering to the flask, the medium was changed every 2 days and the cells were digested using $0.25 \%$ trypsin (Gibco; Thermo Fisher Scientific, Inc.).

MTT assay. Cells were seeded in a 96-well culture plate at a density of $1 \times 10^{4}$ cells/well overnight, and treated with various concentrations of lycorine $(0.2,0.5,1,2,10,20$ and $100 \mu \mathrm{M})$ the following day. Following incubation in a $5 \% \mathrm{CO}_{2}$ incubator at $37^{\circ} \mathrm{C}$ for $24 \mathrm{~h}$ or $48 \mathrm{~h}$, the medium was removed and $20 \mu \mathrm{l}$ MTT solution $(5 \mathrm{mg} / \mathrm{ml})$ was added to each well. Following incubation at $37^{\circ} \mathrm{C}$ for an additional $4 \mathrm{~h}, 150 \mu \mathrm{l}$ dimethyl sulfoxide (Sigma-Aldrich; Merck KGaA, Darmstadt, Germany) was used to dissolve the dark blue crystals. The optical density value was determined at $570 \mathrm{~nm}$ and measured on a microplate reader (Varioskan Flash; Thermo Fisher Scientific, Inc.). All these results are expressed as a percentage of the control, which was set at $100 \%$. Each experiment was repeated three times individually.

Clone formation assay. Cells (200 cells/well) were seeded in a 6-well plate. Cells were allowed to attach overnight and exposed to different concentrations of lycorine (10 and $20 \mu \mathrm{M})$ for $48 \mathrm{~h}$, following which the culture medium was replaced with fresh DMEM and cultured for 2 weeks; cells were subsequently fixed with $4 \%$ paraformaldehyde for $15 \mathrm{~min}$ and stained with $0.1 \%$ crystal violet for $10 \mathrm{~min}$ at room temperature. The number of colonies was counted using Photoshop CS6 software (Adobe Systems, Inc., San Jose, CA, USA). Each group had three repeat wells and this experiment was repeated three times.

Flow cytometry. The cell cycle distribution was determined by flow cytometry. Cells were seeded in a 6-well culture plate at a density of $1 \times 10^{6}$ cells/well. Lycorine $(10$ and $20 \mu \mathrm{M})$ were added the subsequent day. Following incubation for $48 \mathrm{~h}$, cells were harvested and washed twice with PBS. Cells were fixed in cold $75 \%$ ethanol overnight in $4^{\circ} \mathrm{C}$. Cells were washed twice with cold PBS and suspended in PBS with $200 \mu \mathrm{g} / \mathrm{ml}$ RNase and $50 \mu \mathrm{g} / \mathrm{ml}$ propidium iodide (cat. no. 556547; BD Biosciences, San Jose, CA, USA) in the dark for $30 \mathrm{~min}$. The results were measured by flow cytometry (FACScan; BD Biosciences) and analyzed using ModFit LT 3.2 software (Verity Software House, Inc., Topsham, ME, USA).

Wound healing assay. A wound healing assay was used to assess the migration ability of HepG2 cells. Briefly, cells were seeded in a 6-well culture plate. When cells reached $90 \%$ confluence, a wound was scratched with a $200 \mu$ l pipette tip. Cells were washed with PBS three times to remove the scratched cells, and lycorine (10 and $20 \mu \mathrm{M})$ was added and incubated for 24 or $48 \mathrm{~h}$. The cells were imaged following replacement of the medium. The wound width was measured using ImageJ software (version 1.48; National Institutes of Health, Bethesda, MD, USA): Wound healing rate $(\%)=100 \times(0 \mathrm{~h}$ width $-24 / 48 \mathrm{~h}$ width)/2/0 h width.

Transwell assay. HepG2 cells were adjusted to a density of $2 \times 10^{4}$ cells/well, resuspended and seeded in the upper chamber of Transwell chambers with $200 \mu \mathrm{l}$ serum-free medium, and $600 \mu \mathrm{l}$ complete medium containing $30 \%$ FBS was added in the lower chamber. Lycorine (10 and $20 \mu \mathrm{M})$ was added to the two chambers. Following incubation for $48 \mathrm{~h}$, non-migrated cells on the top surface of the upper chamber were gently scraped away with a cotton swab. The lower membrane containing migrated cells was fixed in $4 \%$ paraformaldehyde for $10 \mathrm{~min}$ and stained with $0.1 \%$ crystal violet for $15 \mathrm{~min}$ at room temperature. A microscope (x4 magnification; Olympus IX51; Olympus Corporation, Tokyo, Japan) was used to image the migrated cells. The total cell numbers were calculated from three different fields and three independent experiments.

Western blot analysis. Cells were harvested and lysed in lysis buffer containing $1 \mathrm{mM}$ phenylmethylsulfonyl fluoride (Beyotime Institute of Biotechnology, Haimen, China). Bicinchoninic acid protein quantification kits (Beyotime Institute of Biotechnology) were used to measure protein concentrations. A total of $15 \mu \mathrm{g}$ protein was separated via $12 \%$ SDS-PAGE and transferred to polyvinylidene difluoride membranes (EMD Millipore, Billerica, MA, USA). Following blocking with 5\% skim milk in Tris-buffered saline (TBS) containing $0.1 \%$ Tween-20 for $2 \mathrm{~h}$ at room temperature, specific primary antibodies were added to the membranes 
A

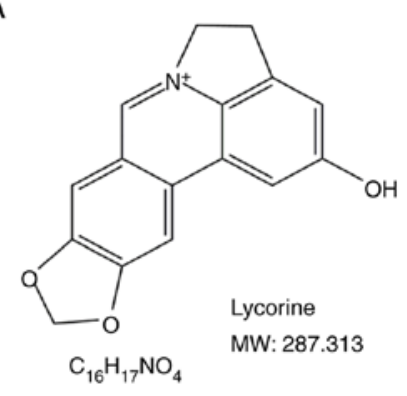

B

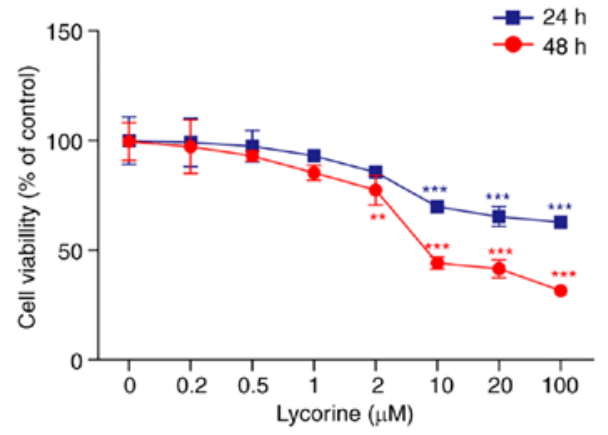

C

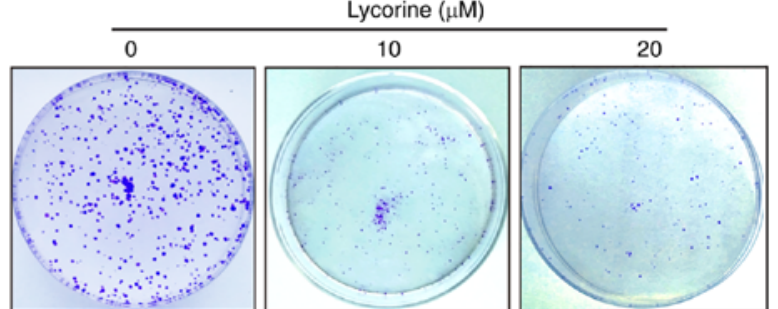

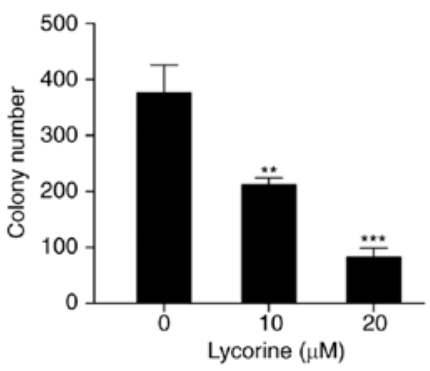

Figure 1. Lycorine inhibits HepG2 cell proliferation. (A) Chemical structure and molecular weight of lycorine. (B) An MTT assay was performed to determine the effect of lycorine on cell viability in HepG2 cells. (C) The effect of lycorine on colony formation in HepG2 cells. Cells were treated with lycorine $(10$ and $20 \mu \mathrm{M})$ for $48 \mathrm{~h}$ and subsequently cultured for 2 weeks. Cells were fixed and stained with crystal violet. The number of colonies was counted and all images are representative of three independent experiments. Data are presented as the mean \pm standard deviation. ${ }^{* *} \mathrm{P}<0.01$ and ${ }^{* * * *} \mathrm{P}<0.001$ vs. control.

and incubated at $4^{\circ} \mathrm{C}$ overnight on a shaker. Membranes were washed in TBS with Tween-20 three times and incubated with anti-rabbit or anti-mouse horseradish peroxidase secondary antibodies (cat. nos. 074-1516 and 074-1802, respectively; 1:100,000; Kirkegaard \& Perry Laboratories Inc., Gaithersburg, MD, USA) for a further $2 \mathrm{~h}$ at room temperature. Enhanced chemiluminescence reagent (EMD Millipore) was used to visualize the bands. Densitometric analysis was performed using Quantity One software version 4.6.2 (Bio-Rad Laboratories, Inc., Hercules, CA, USA). GAPDH was used as an internal control.

Immunofluorescence assay. Following treatment with lycorine $(10$ and $20 \mu \mathrm{M})$ for $48 \mathrm{~h}$, cells were washed twice with PBS and fixed with ice-cold $75 \%$ ethanol for $15 \mathrm{~min}$ at room temperature. Cells were permeabilized with $0.1 \%$ Triton X-100 for $5 \mathrm{~min}$, fluorescent staining of filamentous and globular actin was performed by staining with fluorescent deoxyribonuclease I conjugates and fluorescent phallotoxins (Molecular Probes; Thermo Fisher Scientific, Inc.) for $30 \mathrm{~min}$ in the dark, and slides were washed and stained with DAPI for 5 min at room temperature (cat. no. C1002; Beyotime Institute of Biotechnology). Images were captured using a Leica scanning confocal microscope (x40 magnification; TCS SP2 AOB; Leica Microsystems GmbH, Wetzlar, Germany).

Statistical analysis. All data were analyzed using GraphPad Prism 6.0 software (GraphPad Software, Inc., San Diego, CA, USA). Data presented are expressed as the mean \pm standard deviation at least three independent experiments. Differences between groups were analyzed by one-way analysis of variance (ANOVA) with Dunnett's or Tukey's test. $\mathrm{P}<0.05$ was considered to indicate a statistically significant difference.

\section{Results}

Lycorine inhibits the proliferation of HepG2 cells. The chemical structure and molecular weight of lycorine is presented in Fig. 1A. The cytotoxicity of lycorine in HepG2 cells was determined by MTT assay. Cells were treated with various concentrations of lycorine $(0.2,0.5,1,2,10,20$ and $100 \mu \mathrm{M}$ ) for 24 or $48 \mathrm{~h}$, which resulted in significant decreases in cell viability in a dose-dependent manner $(\mathrm{P}<0.01, \mathrm{P}<0.001$; Fig. 1B). Exposure of cells to $2 \mu \mathrm{M}$ lycorine resulted in a modest decrease in cell viability, and these events became significant following exposure of cells to $\geq 10 \mu \mathrm{M}$ lycorine (Fig. 1B). To further confirm the anti-proliferative effect of lycorine, a clone formation assay was performed. In accordance with the results of the MTT assay, lycorine significantly inhibited the clone formation of HepG2 cells compared with the control cells ( $\mathrm{P}<0.01,10 \mu \mathrm{M} ; \mathrm{P}<0.001,20 \mu \mathrm{M}$; Fig. 1C). Taken together, these results demonstrated that lycorine inhibited the proliferation of HepG2 cells.

Lycorine induces HepG2 cell cycle arrest at the G2/M phase via downregulation of cyclin A, cyclin B1 and cdc2. Cell cycle arrest is an important mechanism involved in the inhibition of cell growth (12). To examine the effect of lycorine on the cell cycle of HepG2 cells, cell cycle dynamics were assessed by flow cytometry. Following exposure of cells to lycorine (10 and $20 \mu \mathrm{M}$ ) for $48 \mathrm{~h}$, the proportion of cells in the $\mathrm{G} 2 / \mathrm{M}$ phase increased from $10.81 \%$ (without lycorine) to $14.64 \%$ (in the presence of $10 \mu \mathrm{M}$ lycorine) and $20.15 \%$ (in the presence of $20 \mu \mathrm{M}$ lycorine; $\mathrm{P}<0.01$ vs. control; Fig. 2A). These results indicated that lycorine induced HepG2 cell cycle arrest at the G2/M phase. To further examine the molecular mechanisms under lycorine-induced $\mathrm{G} 2 / \mathrm{M}$ phase arrest, western blot 
A
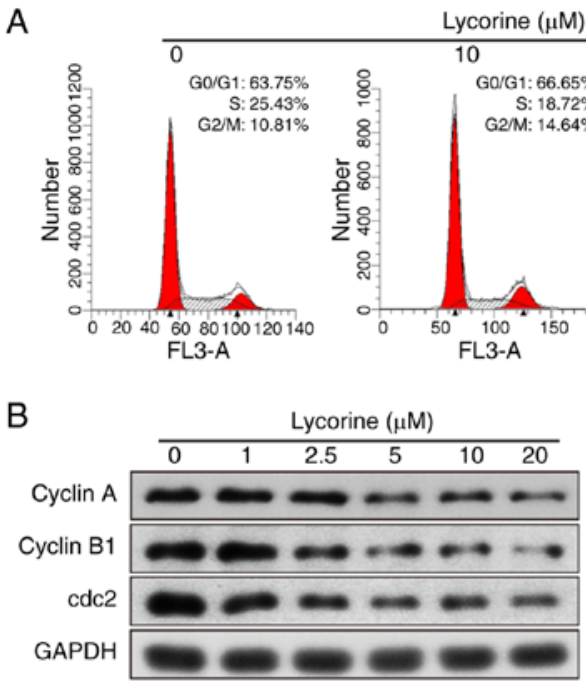
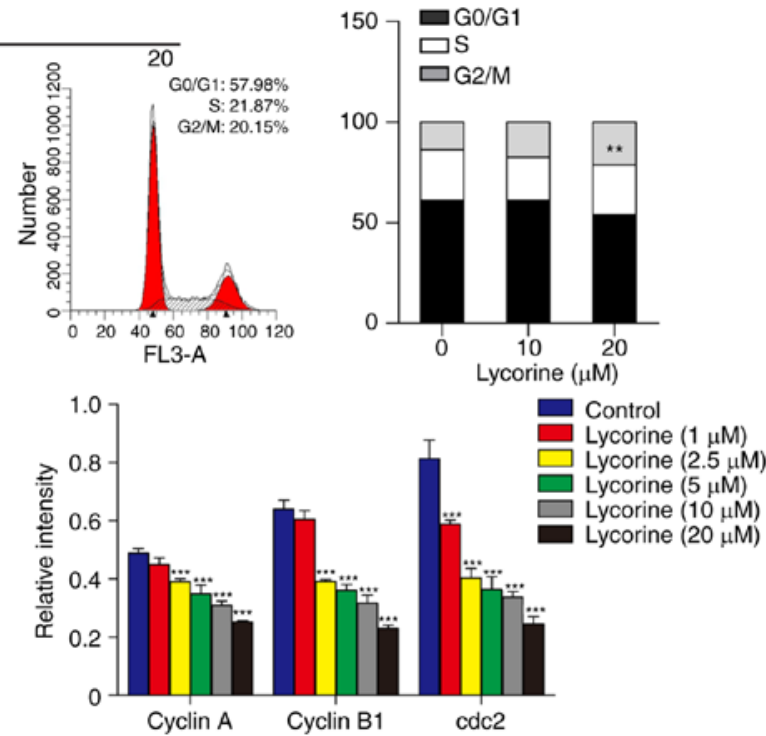

Figure 2. Lycorine induces cell cycle arrest at the G2/M phase in HepG2 cells. (A) The cell cycle was analyzed by flow cytometry following treatment with lycorine $(10$ and $20 \mu \mathrm{M})$ for $48 \mathrm{~h}$. The relative ratios of the G0/G1, S and G2/M phases were analyzed using flow cytometry by ModFit LT 3.2 software. (B) Western blot analysis of the cellular G2/M phase regulators cyclin A, cyclin B1 and cdc2 under treatment with lycorine $(1,2.5,5,10$ and $20 \mu \mathrm{M})$ for $48 \mathrm{~h}$. GAPDH served as the loading control and the relative quantification of the detected proteins was analyzed using Quantity One software. Data are presented as the mean \pm standard deviation. ${ }^{* *} \mathrm{P}<0.01$ and ${ }^{* * * *} \mathrm{P}<0.001$ vs. control. cdc2, cyclin-dependent kinase 1 .

A
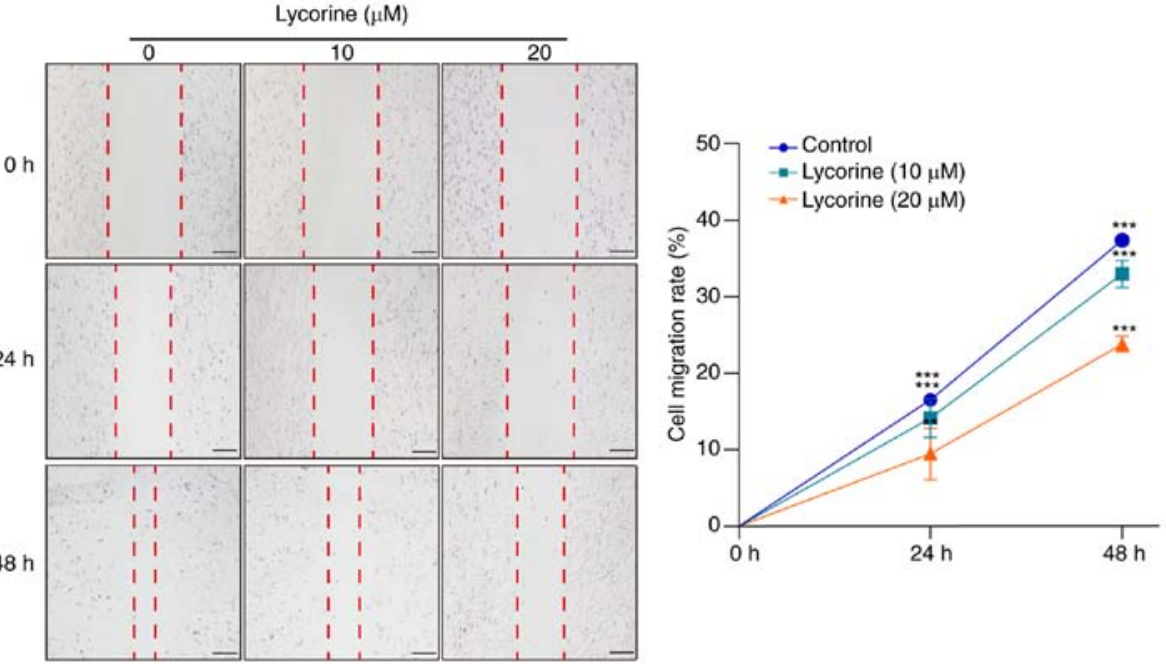

B
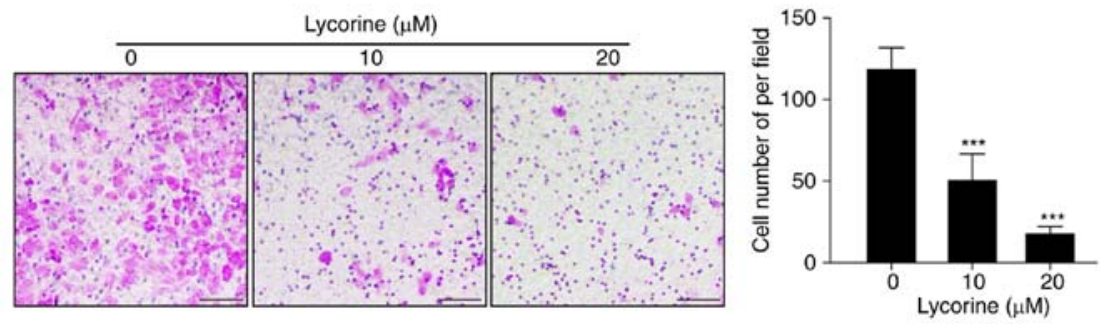

C
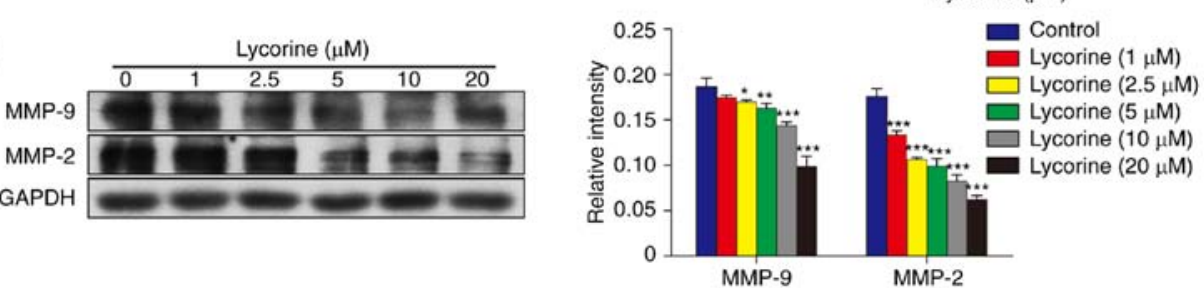

Figure 3. Lycorine inhibits the migration of HepG2 cells. (A) Cells were exposed to serial concentrations of lycorine (10 and $20 \mu \mathrm{M})$ for 24 or $48 \mathrm{~h}$. The effects of lycorine on HepG2 cell migration were evaluated by wound healing assay. Scale bar, $200 \mu \mathrm{m}$. (B) The effect of lycorine on the migration of HepG2 cells was assessed in a Transwell assay. HepG2 cells were treated with lycorine at 10 or $20 \mu \mathrm{M}$ for $48 \mathrm{~h}$. Scale bar, $200 \mu \mathrm{m}$. (C) Following treatment of HepG2 cells with lycorine $(1,2.5,5,10$ and $20 \mu \mathrm{M})$ for $48 \mathrm{~h}$, western blotting was performed to assess the expression of MMP-9 and MMP-2. The relative quantification of proteins was analyzed using Quantity One software. Data are presented the mean \pm standard deviation $(\mathrm{n}=3) .{ }^{*} \mathrm{P}<0.05,{ }^{* * *} \mathrm{P}<0.01$ and ${ }^{* * * *} \mathrm{P}<0.001$ vs. control. MMP, matrix metalloproteinase. 
A
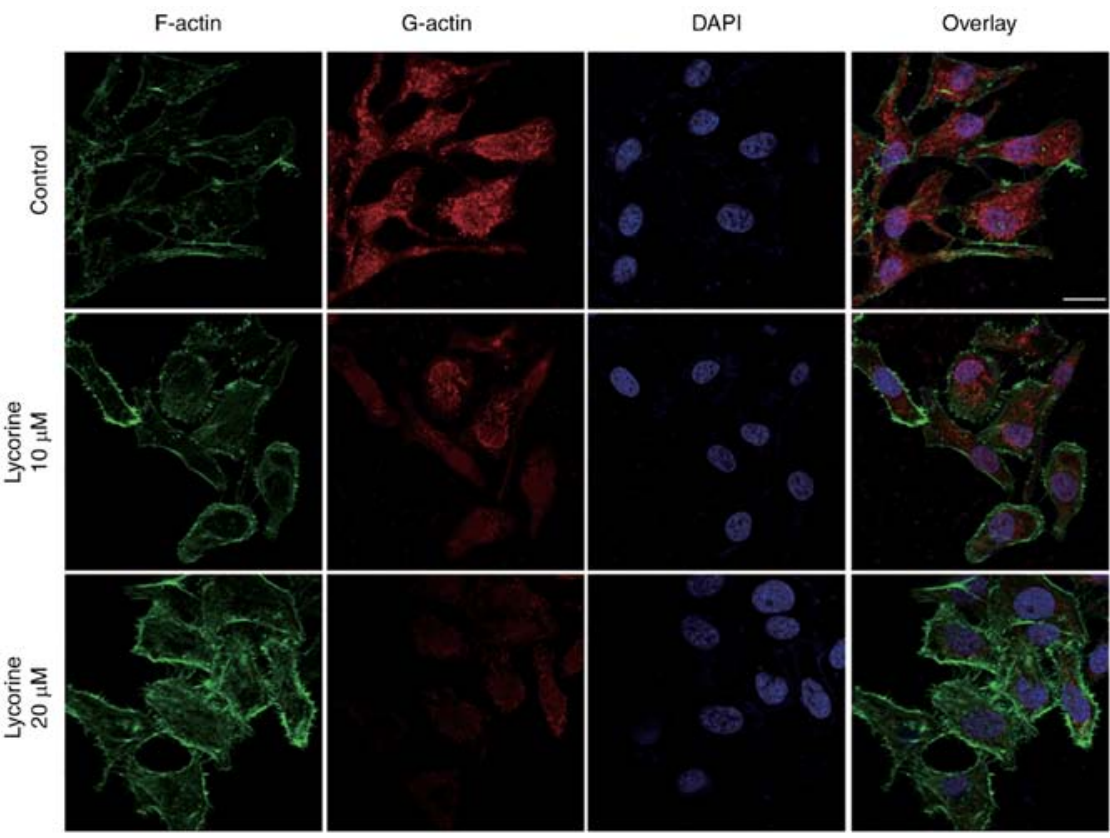

B
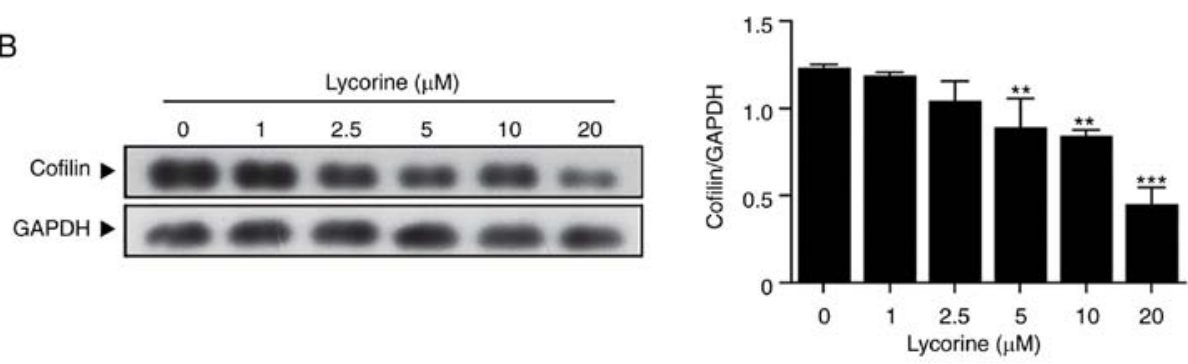

Figure 4. Lycorine induces altering of actin cytoskeletal dynamics by suppressing the expression of cofilin. (A) Levels of F-actin (green), G-actin (red) and DAPI (blue) in cells treated with lycorine $(10$ and $20 \mu \mathrm{M})$ for $48 \mathrm{~h}$ were imaged by confocal microscopy. Scale bar, $20 \mu \mathrm{m}$. (B) Western blotting was used to assess the expression of cofilin. The relative quantification of proteins was analyzed by Quantity One software. Data are presented the mean \pm standard deviation $(\mathrm{n}=3) .{ }^{* *} \mathrm{P}<0.01$ and ${ }^{* * *} \mathrm{P}<0.001$ vs. control.

analysis was performed to assess the expression of cyclin A, cyclin B1 and cdc2. The results revealed that exposure of cells to lycorine resulted in a marked decrease in the expression of cyclin A, cyclin B1 and cdc 2 in HepG2 cells $(\mathrm{P}<0.001$; Fig. 2B). These data suggested that lycorine has an inhibitory effect on HepG2 cell cycle progression, which may cause the inhibition of HepG2 cell proliferation.

Lycorine inhibits the migration of HepG2 cells. The wound healing assay indicated that lycorine induced a marked decrease in cell migration in HepG2 cells in a dose and time-dependent manner $(\mathrm{P}<0.01$ and $\mathrm{P}<0.001$; Fig. 3A). Furthermore, the Transwell assay revealed that lycorine significantly inhibited the migratory ability of HepG2 cells $(\mathrm{P}<0.001$; Fig. 3B). Evidence has confirmed that MMPs, particularly MMP-9 and MMP-2, are important regulators in the process of cancer migration $(13,14)$. To investigate whether lycorine alters the expression of MMP-9 and MMP-2, western blot analysis was performed. The results demonstrated that compared with the control group, lycorine decreased the expression levels of MMP-9 and MMP-2 in a concentration-dependent manner $(\mathrm{P}<0.05, \mathrm{P}<0.01$ and $\mathrm{P}<0.001$; Fig. 3C). These data indicated that lycorine inhibits HepG2 cell migration.
Lycorine alters actin cytoskeletal dynamics by suppressing cofilin expression. Actin, an essential component of the cytoskeleton, has a critical role in a wide range of cellular processes, including cell migration and cell division (15). In the current study, exposure of cells to lycorine (10 and $20 \mu \mathrm{M})$ resulted in an increase in polymerized filamentous actin (F-actin) and a decrease in depolymerized globular actin (G-actin; Fig. 4A). It has been reported that cofilin regulates actin dynamics by severing actin filaments (16). Therefore, western blot analysis was performed to determine whether cofilin is involved in the lycorine-induced altering of actin cytoskeletal dynamics. As presented in Fig. 4B, HepG2 cells were treated with lycorine $(1,2.5,5,10$ and $20 \mu \mathrm{M})$ for $48 \mathrm{~h}$, which resulted in a decrease in the expression of cofilin $(\mathrm{P}<0.01$ and $\mathrm{P}<0.001)$. Collectively, these results suggested that lycorine suppresses the expression of cofilin and alters actin cytoskeletal dynamics.

ROCK1 activation has an important role in lycorine-induced anti-proliferative and anti-migration effects. ROCK1 has been confirmed to have an important role in regulating cell polarity and migration $(17,18)$. In the present study, it was investigated whether ROCK1 activation is involved in lycorine-induced anticancer effects. Treatment of HepG2 cells with lycorine $(1,2.5,5,10$ and $20 \mu \mathrm{M})$ for $48 \mathrm{~h}$ 
A

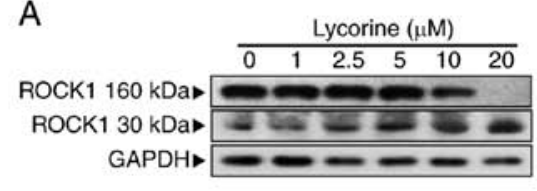

C

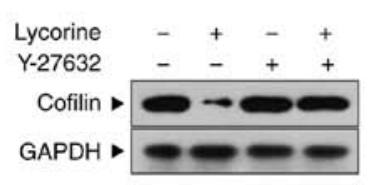

D

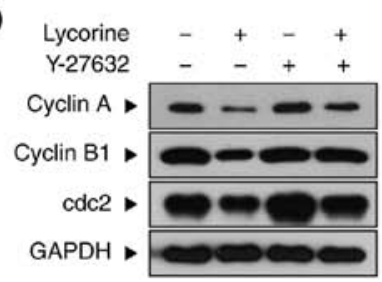

$\mathrm{F}$

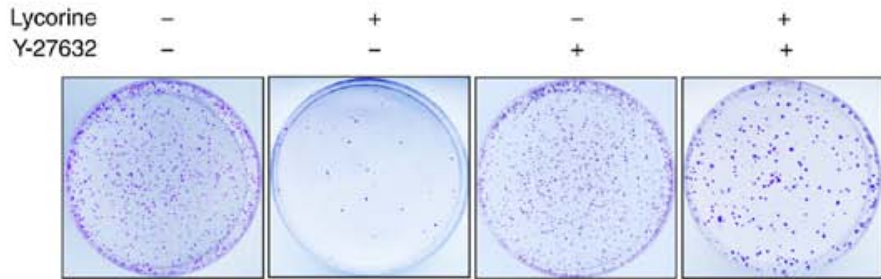

G Lycorine Y-27632

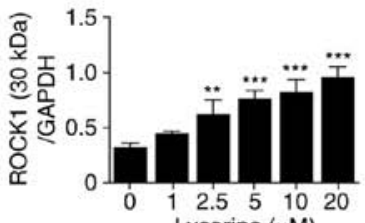

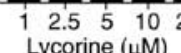

$E$
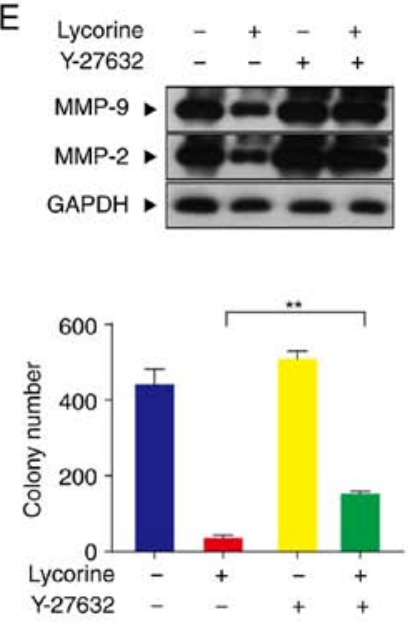

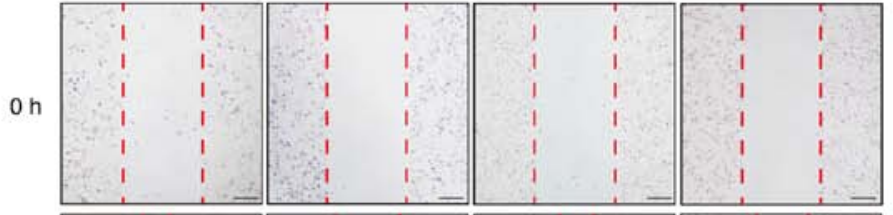

$48 \mathrm{~h}$

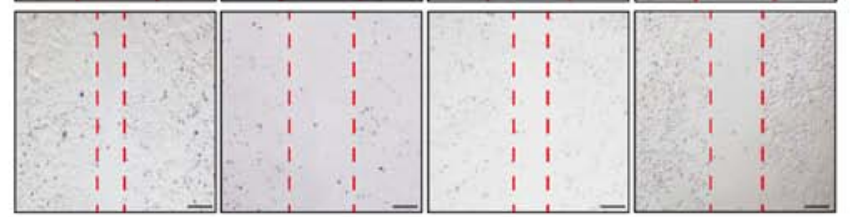

$\mathrm{H}$
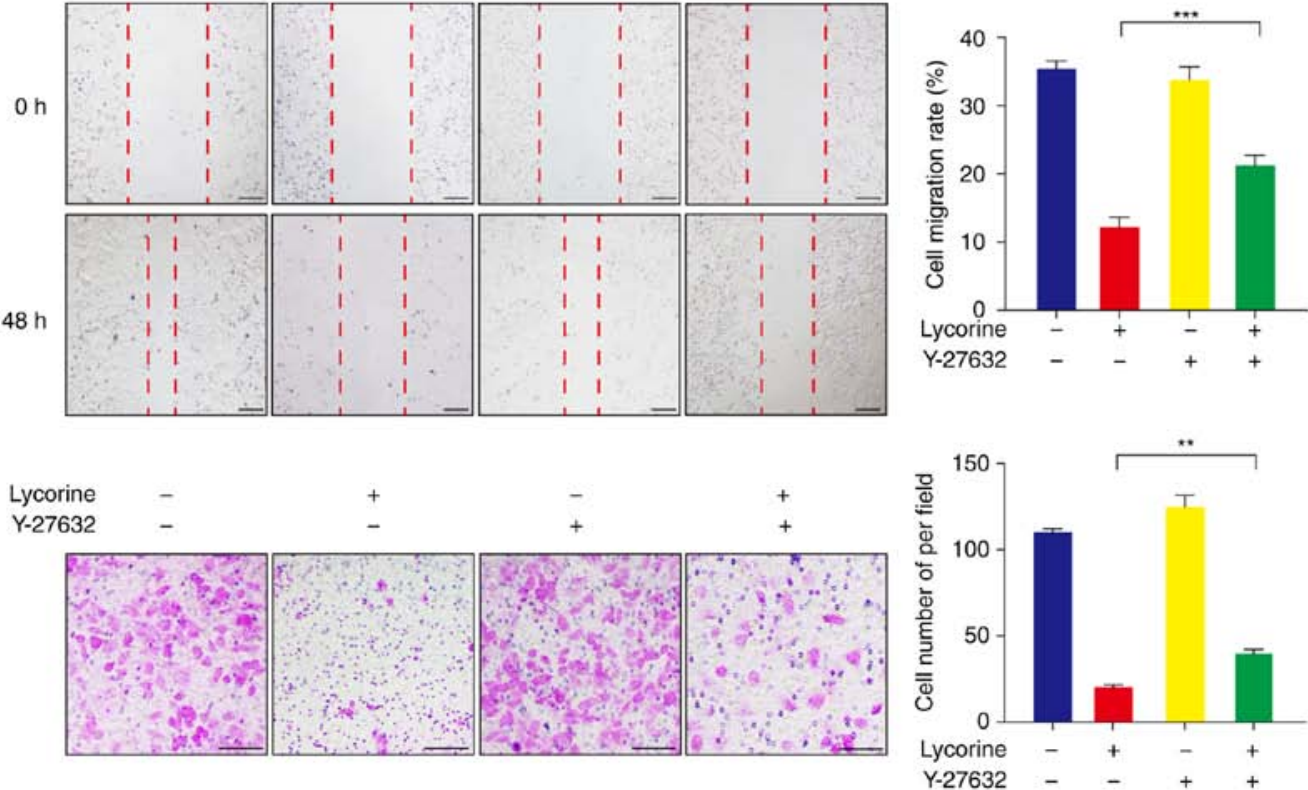

Figure 5. ROCK1 activation has an important role in lycorine-induced anti-proliferative and anti-migration effects. (A) Cells were treated with lycorine (1, 2.5, 5,10 and $20 \mu \mathrm{M}$ ) for $48 \mathrm{~h}$ and western blot analysis was used to assess the expression levels of ROCK1 (160 kDa) and cleaved ROCK1 (30 kDa). The relative quantification of proteins was analyzed using Quantity One software. Data are presented the mean $\pm \mathrm{SD}(\mathrm{n}=3)$. ${ }^{* *} \mathrm{P}<0.01$ and ${ }^{* * *} \mathrm{P}<0.001$ vs. control. $(\mathrm{B}) \mathrm{Cells}$ were pretreated with Y-27632 $(20 \mu \mathrm{M})$, a specific ROCK1 inhibitor, for $2 \mathrm{~h}$, followed by treating with lycorine $(20 \mu \mathrm{M})$ for $48 \mathrm{~h}$. Western blotting was used to assess the expression of ROCK1 (160 kDa) and cleaved ROCK1 (30 kDa). (C) Following preincubation of cells with Y-27632, western blotting was used to assess the expression of cofilin. (D) Following preincubation of cells with Y-27632, western blotting was used to assess the expression of cyclin A, cyclin B1 and cdc2. (E) Following preincubation of cells with Y-27632, western blotting was used to assess the expression of MMP-9 and MMP-2. (F) Cells were fixed and stained with crystal violet, the number of cell colonies was counted. (G) Cell migration was measured by wound healing assay and results are presented graphically. Scale bar, $200 \mu \mathrm{m}$. (H) Cell migration was measured in a Transwell assay. Scale bar, $200 \mu \mathrm{m}$. Data are presented the mean $\pm \mathrm{SD}(\mathrm{n}=3)$. ${ }^{* *} \mathrm{P}<0.01$ and ${ }^{* * *} \mathrm{P}<0.001$ vs. the lyorine-only treatment group. ROCK1, Rho associated coiled-coil containing protein kinase 1 ; SD, standard deviation; MMP, matrix metalloproteinase; cdc2, cyclin dependent kinase 1.

induced cleavage/activation of ROCK1 in a dose-dependent manner (Fig. 5A). To further confirm the role of ROCK1 in lycorine-induced cell proliferation and migration inhibition, Y-27632, a ROCK1 specific inhibitor, was used. Western blot analysis indicated that pre-incubation of cells with
Y-27632 inhibited lycorine-induced ROCK1 cleavage/activation (Fig. 5B). Pre-incubation of cells with Y-27632 attenuated the lycorine-induced cofilin decrease (Fig. 5C). Furthermore, pre-incubation with Y-27632 also attenuated the decreases in cyclin A, cyclin B1, cdc2, MMP-9 and MMP-2, which indicated 
that Y-27632 attenuated lycorine-induced G2/M cell cycle arrest and migration ability (Fig. 5D and E). A clone formation assay demonstrated that combined treatment with Y-27632 and lycorine significantly increased the colony number compared with treatment with lycorine alone $(\mathrm{P}<0.01$; Fig. $5 \mathrm{~F})$. The wound healing and Transwell assays revealed that co-administration of Y-27632 and lycorine markedly attenuated the lycorine-induced inhibitory effect on cell migration $(\mathrm{P}<0.01$, $\mathrm{P}<0.001$; Fig. 5G and H). Collectively, these results demonstrated that ROCK1 activation has a critical role in the lycorine-induced effects on actin cytoskeletal dynamics, and anti-proliferative and anti-migration activity in $\mathrm{HepG} 2$ cells.

\section{Discussion}

In recent years, naturally occurring compound have received increasing attention in cancer research. Lycorine, a natural compound obtained from the Amaryllidaceae plant family, possesses anti-cancer activity in breast cancer, bladder cancer and multiple myeloma $(10,19,20)$. However, the function and associated mechanisms of lycorine have not been examined in hepatoblastoma. In the current study, the inhibitory effects of lycorine on cell proliferation and migration were investigated in HepG2 cells. The HepG2 cell line was originally established in 1979 by Aden et al (21) and mistakenly reported as hepatocellular carcinoma. In recent years, the HepG2 cell line has frequently been used for hepatoblastoma research (3). The results of the present study indicated that lycorine inhibited HepG2 cell proliferation by inducing cell cycle arrest at the $\mathrm{G} 2 / \mathrm{M}$ phase, and decreasing the expression of cyclin A, cyclin $\mathrm{B} 1$ and cdc2. In addition, lycorine decreased the migration ability of HepG2 cells. Furthermore, lycorine altered actin cytoskeletal dynamics by suppressing the expression of cofilin, and ROCK1 activation was demonstrated to have an important role in the anti-proliferative and anti-migration effects of lycorine in HepG2 hepatoblastoma cells.

Agents that possess the ability to inhibit the proliferation and migration of tumor cells may be used to inhibit cancer progression and increase survival rates (22). In the present study, treatment with lycorine effectively inhibited the cell proliferation and colony formation of HepG2 cells. It has been previously reported that lycorine induces cell cycle arrest at the G0/G1 phase in K562 cells (23) and KM3 cells (24). However, in the current study, lycorine induced HepG2 cell cycle arrest at the G2/M phase in a dose-dependent manner. The cell cycle is a physiological process, including the G0/G1, $\mathrm{S}$ and $\mathrm{G} 2 / \mathrm{M}$ phases. A series of proteins, including cyclins and cyclin-dependent kinases, regulate the cell cycle (25). In the present study, cyclin A, cyclin B1 and cdc2 were significantly downregulated in HepG2 cells following treatment with lycorine. These findings suggested that lycorine induced cell cycle arrest at the G2/M phase via inhibition of cyclin A, cyclin B1 and cdc2 expression in HepG2 cells.

Metastasis is considered to be the primary cause of mortality in the majority of patients with cancer. The migration and invasion ability of tumor cells are key factors in tumor metastasis. Previous studies have demonstrated that lycorine inhibits the growth and metastasis of breast cancer through inhibition of signal transducer and activator of transcription 3 signaling (19). Similarly, in the current study, lycorine inhibited the migration of HepG2 cells. The MMP family, generally considered to be biomarkers for cancers, are reportedly upregulated in the majority of types of cancer (26). Furthermore, MMPs have also been considered to be potential therapeutic targets in cancer (27-29). In particular, MMP-9 and MMP-2, exhibiting enzymatic collagenase activity, are typical members among them (30). ROCK1, which serves an important role in cell polarity and migration, regulates the expression of MMP-9 and MMP-2 (31,32). Jeong et al (33) demonstrated that lysophosphatidic acid increases ovarian cancer cell invasion via a Ras/Rho/ROCK signaling pathway and subsequent production of the proteolytic enzyme MMP-9. Cofilin is an important downstream mediator of ROCK1, and the present study revealed that inhibition of the ROCK1/cofilin pathway decreased the expression of MMP-9 and MMP-2. These results indicated that the anti-migration effects of lycorine on HepG2 cells may be associated with the lycorine-induced downregulation of MMP-9 and MMP-2.

Previous studies have indicated that the reorganization of the actin cytoskeleton is the basis of cancer cell migration, adhesion and invasion (34-36). The current findings demonstrated that lycorine blocked the normal dynamic turnover of the actin cytoskeleton, with an increase in polymerized F-actin and a loss of depolymerized G-actin. A number of actin-binding proteins have been reported to be involved in the regulation of actin dynamics. For example, cofilin, a member of the actin depolymerizing factor/cofilin family, exerts its effects on actin filament dynamics by binding to F-actin and severing actin filaments. Cofilin has been reported to be overexpressed in pancreatic cancer cells, A549 lung cancer cells and the rat C6 glioblastoma cell line $(14,37,38)$. Furthermore, Yap et al (39) reported that overexpression of cofilin enhanced cell motility in U373 astrocytoma cells. In the present study, treatment with lycorine decreased the expression of cofilin in a dose-dependent manner. ROCK1, an upstream regulator of cofilin, has an important role in regulating cell polarity and migration $(34,40-42)$. Furthermore, Y-27632, a ROCK1 specific inhibitor, stimulates proliferation in various cell lines (43-45), indicating that the activation of ROCK1 inhibits cell proliferation. In the present study, ROCK1 activation was associated with lycorine-induced anti-proliferative and anti-migration effects in HepG2 cells. Treatment with lycorine resulted in the cleavage/activation of ROCK1, and pre-incubation of cells with Y-27632 blocked lycorine-induced decreases in cofilin, cyclin A, cyclin B1, cdc2, MMP-9 and MMP-2. Furthermore, combined treatment with Y-27632 and lycorine markedly attenuated lycorine-induced clone formation inhibition and its inhibitory effects on migratory ability. Taken together, these results demonstrated that lycorine alters actin cytoskeletal dynamics by suppressing the expression of cofilin and activating ROCK1.

In conclusion, the data demonstrated that lycorine inhibited HepG2 hepatoblastoma cell proliferation and migration through inhibition of ROCK1/cofilin-induced actin dynamics. All of the findings provide support for the development of lycorine as a potential drug candidate for anti-hepatoblastoma therapy.

\section{Acknowledgements}

Not applicable. 


\section{Funding}

The present study was supported by grants from the National Natural Science Foundation of China (grant no. 31600806), Clinical Research Projects of Xinqiao Hospital, Army Medical University (grant no. 2016YLC12), and the Foundation of Chongqing Science and Technology Commission (grant no. CSTC2015SHMSZX120078).

\section{Availability of data and materials}

The datasets used and/or analyzed during the present study are available from the corresponding author on reasonable request.

\section{Authors' contributions}

WL, RZ and GL conceived and designed the study. WL, QZ, QT and $\mathrm{CH}$ performed the experiments. JH, YLi, YLu and QW analyzed the data. WL and RZ wrote the manuscript. GL and QZ reviewed and edited the manuscript. All authors read and approved the manuscript and agree to be accountable for all aspects of the research in ensuring that the accuracy or integrity of any part of the report work are appropriately investigated and resolved.

\section{Ethics approval and consent to participate}

Not applicable.

\section{Patient consent for publication}

Not applicable.

\section{Competing interests}

The authors declare that they have no competing interests.

\section{References}

1. Zsiros J, Maibach R, Shafford E, Brugieres L, Brock P, Czauderna P, Roebuck D, Childs M, Zimmermann A, Laithier V, et al: Successful treatment of childhood high-risk hepatoblastoma with dose-intensive multiagent chemotherapy and surgery: Final results of the SIOPEL-3HR study. J Clin Oncol 28: 2584-2590, 2010.

2. Zhang YT, Feng LH, Zhong XD, Wang LZ and Chang J: Single-agent cisplatin treatment of children with high-risk hepatoblastoma. J Pediatr Hematol Oncol 36: 271-275, 2014.

3. Yu Y, Zhao X, Zhang Y, Kang Y, Wang J and Liu Y: Antitumor activity of YM155, a selective survivin suppressant, in combination with cisplatin in hepatoblastoma. Oncol Rep 34: 407-414 2015.

4. Jimenez A, Santos A, Alonso G and Vazquez D: Inhibitors of protein synthesis in eukarytic cells. Comparative effects of some amaryllidaceae alkaloids. Biochim Biophys Acta 425: 342-348, 1976.

5. Toriizuka Y, Kinoshita E, Kogure N, Kitajima M, Ishiyama A, Otoguro K, Yamada H, Omura S and Takayama H: New lycorine-type alkaloid from Lycoris traubii and evaluation of antitrypanosomal and antimalarial activities of lycorine derivatives. Bioorg Med Chem 16: 10182-10189, 2008

6. Shen JW, Ruan Y, Ren W, Ma BJ, Wang XL and Zheng CF: Lycorine: A potential broad-spectrum agent against crop pathogenic fungi. J Microbiol Biotechnol 24: 354-358, 2014.

7. Guo Y, Wang Y, Cao L, Wang P, Qing J, Zheng Q, Shang L, Yin Z and Sun Y: A conserved inhibitory mechanism of a lycorine derivative against enterovirus and hepatitis $\mathrm{C}$ virus. Antimicrob Agents Chemother 60: 913-924, 2016.
8. Lamoral-Theys D, Andolfi A, Van Goietsenoven G, Cimmino A, Le Calvé B, Wauthoz N, Mégalizzi V, Gras T, Bruyère $\mathrm{C}$, Dubois $\mathrm{J}$, et al: Lycorine, the main phenanthridine Amaryllidaceae alkaloid, exhibits significant antitumor activity in cancer cells that display resistance to proapoptotic stimuli: An investigation of structure-activity relationship and mechanistic insight. J Med Chem 52: 6244-6256, 2009.

9. Zeng H, Fu R, Yan L and Huang J: Lycorine induces apoptosis of A549 cells via AMPK-mammalian target of rapamycin (mTOR)-S6K signaling pathway. Med Sci Monit 23: 2035-2041, 2017.

10. Wang C, Wang Q, Li X, Jin Z, Xu P, Xu N, Xu A, Xu Y, Zheng S, Zheng $\mathrm{J}$, et al: Lycorine induces apoptosis of bladder cancer T24 cells by inhibiting phospho-Akt and activating the intrinsic apoptotic cascade. Biochem Biophys Res Commun 483: 197-202, 2017.

11. Cao Z, Yu D, Fu S, Zhang G, Pan Y, Bao M, Tu J, Shang B, Guo P, Yang $\mathrm{P}$, et al: Lycorine hydrochloride selectively inhibits human ovarian cancer cell proliferation and tumor neovascularization with very low toxicity. Toxicol Lett 218: 174-185, 2013.

12. King KL and Cidlowski JA: Cell cycle regulation and apoptosis. Annu Rev Physiol 60: 601-617, 1998.

13. Gialeli C, Theocharis AD and Karamanos NK: Roles of matrix metalloproteinases in cancer progression and their pharmacological targeting. Febs J 278: 16-27, 2011.

14. Sinha P, Hütter G, Köttgen E, Dietel M, Schadendorf D and Lage H: Increased expression of epidermal fatty acid binding protein, cofilin, and 14-3-3-sigma (stratifin) detected by two-dimensional gel electrophoresis, mass spectrometry and microsequencing of drug-resistant human adenocarcinoma of the pancreas. Electrophoresis 20: 2952-2960, 1999.

15. Gourlay CW and Ayscough KR: The actin cytoskeleton: A key regulator of apoptosis and ageing? Nat Rev Mol Cell Biol 6: 583-589, 2005.

16. Gross SR: Actin binding proteins: Their ups and downs in metastatic life. Cell Adh Migr 7: 199-213, 2013.

17. Ding W, Tan H, Zhao C, Li X, Li Z, Jiang C, Zhang Y and Wang L: MiR-145 suppresses cell proliferation and motility by inhibiting ROCK1 in hepatocellular carcinoma. Tumour Biol 37: 6255-6260, 2016.

18. Genda T, Sakamoto M, Ichida T, Asakura H, Kojiro M, Narumiya $S$ and Hirohashi S: Cell motility mediated by rho and Rho-associated protein kinase plays a critical role in intrahepatic metastasis of human hepatocellular carcinoma. Hepatology 30: 1027-1036, 1999.

19. Wang J, Xu J and Xing G: Lycorine inhibits the growth and metastasis of breast cancer through the blockage of STAT3 signaling pathway. Acta Biochim Biophys Sin 49: 771-779, 2017.

20. Roy M, Liang L, Xiao X, Peng Y, Luo Y, Zhou W, Zhang J, Qiu L, Zhang S, Liu F, et al: Lycorine downregulates HMGB1 to inhibit autophagy and enhances bortezomib activity in multiple myeloma. Theranostics 6: 2209-2224, 2016.

21. Aden DP, Fogel A, Plotkin S, Damjanov I and Knowles BB: Controlled synthesis of HBsAg in a differentiated human liver carcinoma-derived cell line. Nature 282: 615-616, 1979.

22. Liu Y, Bi T, Shen G, Li Z, Wu G, Wang Z, Qian L and Gao Q: Lupeol induces apoptosis and inhibits invasion in gallbladder carcinoma GBC-SD cells by suppression of EGFR/MMP-9 signaling pathway. Cytotechnology 68: 123-133, 2016.

23. Li L, Dai HJ, Ye M, Wang SL, Xiao XJ, Zheng J, Chen HY, Luo YH and Liu J: Lycorine induces cell-cycle arrest in the G0/G1 phase in K562 cells via HDAC inhibition. Cancer Cell Int 12: 49, 2012.

24. Li Y, Liu J, Tang LJ, Shi YW, Ren W and Hu WX: Apoptosis induced by lycorine in KM3 cells is associated with the G0/G1 cell cycle arrest. Oncol Rep 17: 377-384, 2007.

25. Vermeulen K, Van Bockstaele DR and Berneman ZN: The cell cycle: A review of regulation, deregulation and therapeutic targets in cancer. Cell Prolif 36: 131-149, 2003.

26. Benson CS, Babu SD, Radhakrishna S, Selvamurugan N and Ravi Sankar B: Expression of matrix metalloproteinases in human breast cancer tissues. Dis Markers 34: 395-405, 2013.

27. Roy R, Yang J and Moses MA: Matrix metalloproteinases as novel biomarkers and potential therapeutic targets in human cancer. J Clin Oncol 27: 5287-5297, 2009.

28. Komiya Y, Kurabe N, Katagiri K, Ogawa M, Sugiyama A, Kawasaki Y and Tashiro F: A novel binding factor of 14-3-3beta functions as a transcriptional repressor and promotes anchorage-independent growth, tumorigenicity, and metastasis. J Biol Chem 283: 18753-18764, 2008. 


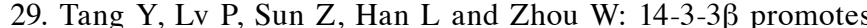
migration and invasion of human hepatocellular carcinoma cells by modulating expression of MMP2 and MMP9 through PI3K/Akt/NF-kappaB pathway. PLoS One 11: e0146070, 2016.

30. Mendes O, Kim HT and Stoica G: Expression of MMP2, MMP9 and MMP3 in breast cancer brain metastasis in a rat model. Clin Exp Metastasis 22: 237-246, 2005.

31. Schram K, Ganguly R, No EK, Fang X, Thong FS and Sweeney G: Regulation of MT1-MMP and MMP-2 by leptin in cardiac fibroblasts involves Rho/ROCK-dependent actin cytoskeletal reorganization and leads to enhanced cell migration. Endocrinology 152: 2037-2047, 2011.

32. Liu X, Chen D and Liu G: Overexpression of RhoA promotes the proliferation and migration of cervical cancer cells. Biosci Biotechnol Biochem 78: 1895-1901, 2014

33. Jeong KJ, Park SY, Cho KH, Sohn JS, Lee J, Kim YK, Kang J, Park CG, Han JW and Lee HY: The Rho/ROCK pathway for lysophosphatidic acid-induced proteolytic enzyme expression and ovarian cancer cell invasion. Oncogene 31: 4279-4289, 2012.

34. Ishaq M, Lin BR, Bosche M, Zheng X, Yang J, Huang D Lempicki RA, Aguilera-Gutierrez A and Natarajan V: LIM kinase 1-dependent cofilin 1 pathway and actin dynamics mediate nuclear retinoid receptor function in $\mathrm{T}$ lymphocytes. BMC Mol Biol 12: 41, 2011.

35. Flamini MI, Fu XD, Sanchez AM, Giretti MS, Garibaldi S, Goglia L, Pisaneschi S, Tosi V, Genazzani AR and Simoncini T: Effects of raloxifene on breast cancer cell migration and invasion through the actin cytoskeleton. J Cell Mol Med 13: 2396-2407, 2009

36. Freitas VM, Rangel M, Bisson LF, Jaeger RG and Machado-Santelli GM: The geodiamolide $\mathrm{H}$, derived from Brazilian sponge Geodia corticostylifera, regulates actin cytoskeleton, migration and invasion of breast cancer cells cultured in three-dimensional environment. J Cell Physiol 216: 583-594, 2008

37. Keshamouni VG, Michailidis G, Grasso CS, Anthwal S, Strahler JR, Walker A, Arenberg DA, Reddy RC, Akulapalli S, Thannickal VJ, et al: Differential protein expression profiling by iTRAQ-2DLC-MS/MS of lung cancer cells undergoing epithelial-mesenchymal transition reveals a migratory/invasive phenotype. J Proteome Res 5: 1143-1154, 2006.
38. Wang W, Goswami S, Lapidus K, Wells AL, Wyckoff JB, Sahai E, Singer RH, Segall JE and Condeelis JS: Identification and testing of a gene expression signature of invasive carcinoma cells within primary mammary tumors. Cancer Res 64: 8585-8594, 2004.

39. Yap CT, Simpson TI, Pratt T, Price DJ and Maciver SK: The motility of glioblastoma tumour cells is modulated by intracellular cofilin expression in a concentration-dependent manner. Cell Motil Cytoskeleton 60: 153-165, 2005.

40. Salvarezza SB, Deborde S, Schreiner R, Campagne F, Kessels MM, Qualmann B, Caceres A, Kreitzer G and Rodriguez-Boulan E: LIM kinase 1 and cofilin regulate actin filament population required for dynamin-dependent apical carrier fission from the trans-Golgi network. Mol Biol Cell 20: 438-451, 2009.

41. Kaji N, Muramoto A and Mizuno K: LIM kinase-mediated cofilin phosphorylation during mitosis is required for precise spindle positioning. J Biol Chem 283: 4983-4992, 2008.

42. San Martin A, Lee MY, Williams HC, Mizuno K, Lassègue B and Griendling KK: Dual regulation of cofilin activity by LIM kinase and Slingshot-1L phosphatase controls platelet-derived growth factor-induced migration of human aortic smooth muscle cells. Circ Res 102: 432-438, 2008.

43. Okumura N, Ueno M, Koizumi N, Sakamoto Y, Hirata K, Hamuro J and Kinoshita S: Enhancement on primate corneal endothelial cell survival in vitro by a ROCK inhibitor. Invest Ophthalmol Vis Sci 50: 3680-3687, 2009.

44. Nakamura K, Yoshimura A, Kaneko T, Sato K and Hara Y: ROCK inhibitor Y-27632 maintains the proliferation of confluent human mesenchymal stem cells. J Periodontal Res 49: 363-370, 2014.

45. Del Debbio CB, Santos MF, Yan CY, Ahmad I and Hamassaki DE: Rho GTPases control ciliary epithelium cells proliferation and progenitor profile induction in vivo. Invest Ophthalmol Vis Sci 55: 2631-2641, 2014 